\title{
PRIMITIVE IDEMPOTENT MEASURES ON COMPACT SEMIGROUPS
}

\author{
by J. DUNCAN \\ (Received 18th June 1969)
}

\section{Introduction}

Let $S$ be a compact semigroup (with jointly continuous multiplication) and let $P(S)$ denote the probability measures on $S$, i.e. the positive regular Borel measures on $S$ with total mass one. Then $P(S)$ is a compact semigroup with convolution multiplication and the weak ${ }^{*}$ topology. Let $\Pi(P(S))$ denote the set of primitive (or minimal) idempotents in $P(S)$. Collins (2) and Pym (5) respectively have given complete descriptions of $\Pi(P(S))$ when $S$ is a group and when $K(S)$, the kernel of $S$, is not a group. Choy (1) has given some characterizations of $\Pi(P(S))$ for the general case. In this paper we present some detailed and intrinsic characterizations of $\Pi(P(S))$ for various classes of compact semigroups that are not covered by the results of Collins and Pym.

In Section 2 we give a detailed survey of the known results on $\Pi(P(S))$ together with some preliminary results. We include here some facts about maximal simple subsemigroups of compact semigroups. In Section $\mathbf{3}$ we consider the commutative case. We show that the elements of $\Pi(P(S))$ are then the Haar measures on certain of the maximal subgroups of $S$ together with the Haar measures on the maximal closed subgroups of $K(S)$. (Throughout this paper, Haar measure means normalized Haar measure.) If $m$ is the Haar measure on $K(S)$, then $\Pi(P(S)) \cup\{m\}$ is a compact idempotent semigroup in which all distinct products are $m$ and the topology is the one point compactification of $\Pi(P(S))$ with the discrete topology. As an amusing application we obtain an identification of $\Pi(P(S))$ with $\Pi(P(P(S)))$. Similar results obtain for compact semigroups $S$ such that each idempotent of $S$ is central and $K(S)$ is commutative. In Section 4 we describe the central primitive idempotents on an arbitrary compact semigroup $S$. Under some weak commutativity assumptions we describe the elements of $\Pi(P(S))$ in terms of the maximal simple subsemigroups of $S$. Some of the results in Section 3 could be deduced as special cases of the results in Section 4, but it seems simpler for the exposition to consider the commutative case first.

Given $\mu \in P(S)$, we write supp $\mu$ for the support of $\mu$, i.e. the unique minimal closed subset of $S$ with $\mu$-mass one. Then for $\mu, v \in P(S)$, we have

$$
\operatorname{supp} \mu \nu=\operatorname{supp} \mu \operatorname{supp} v
$$

and when $\mu$ is idempotent, supp $\mu$ is a simple subsemigroup of $S$ (see Pym (5)). When $S$ is a group we have $\mu^{2}=\mu$ if and only if $\operatorname{supp} \mu$ is a group and $\mu$ is the 
Haar measure on $\operatorname{supp} \mu$. We shall always identify $\mu$ with its restriction to any closed subset of $S$ that contains supp $\mu$. Moreover, given $\mu \in P(E)$, where $E$ is a closed subset of $S$ we also write $\mu$ for the natural extension of $\mu$ to a probability measure on $S$. Given $x \in S$ we write $\delta_{x}$ for the point mass at $x$.

\section{Preliminary results}

Let $T$ be any semigroup and let $I(T)$ denote the set of idempotents of $T$. The natural partial order on $I(T)$ is defined by

$$
e \leqq f \text { if } e f=f e=e .
$$

If $T$ has a zero element $\theta$, then $\theta \leqq e$ for each idempotent $e$. An idempotent $e$ of $T$ is primitive if it is a minimal non-zero element of $(I(T), \leqq)$. The set of all primitive idempotents of $T$ is denoted by $\Pi(T)$. The elementary description of $\Pi(T)$ varies according as $T$ has a zero element or not. The following lemma is elementary.

Lemma 1. Let $T$ be a semigroup and let $e \in I(T)$.

(i) If $T$ has no zero element, then $e \in \Pi(T)$ if and only if $I(e T e)=\{e\}$.

(ii) If $T$ has zero element $\theta$, then $e \in \Pi(T)$ if and only if $I(e T e)=\{e, \theta\}$.

In studying the primitive idempotent measures on a compact semigroup Pym (5) modified the definition somewhat. Given a semigroup $T$, let $T_{\theta}$ be the semigroup obtained by adjoining a zero element $\theta$ to $T$ (whether or not $T$ itself has a zero element). Given $e \in I(T)$, we say that $e \in \Pi^{*}(T)$ if $e \in \Pi\left(T_{\theta}\right)$. (This is Pym's definition of primitive idempotent when $T=P(S)$.) Since $e T_{\theta} e=e T e \cup\{\theta\}$, it follows from Lemma 1 that $e \in \Pi^{*}(T)$ if and only if

$$
I(e T e)=\{e\} .
$$

Thus if $T$ has no zero element then $\Pi^{*}(T)=\Pi(T)$. On the other hand if $T$ has a zero element, say $m$, then $\Pi^{*}(T)=\{m\}$. Now let $T=P(S)$, where $S$ is a compact semigroup. Then $T$ has a zero element if and only if $K(S)$ is a group, in which case the zero element is the Haar measure on $K(S)$. When $K(S)$ is not a group, Pym (5) gives a complete description of

$$
\Pi(P(S))=\Pi^{*}(P(S)) \text {. }
$$

Theorem 2. (Pym) Let $S$ be a compact semigroup whose kernel $K(S)$ is not a group. Then

(i) $\Pi(P(S))=K(P(S))$,

(ii) each $\mu \in \Pi(P(S))$ is supported in $K(S)$; and if $E \times G \times F$ is a canonical decomposition of $K(S)$, then

$$
\mu=\mu_{1} \times m \times \mu_{2}
$$

where $\mu_{1} \in P(E), \mu_{2} \in P(F)$ and $m$ is the Haar measure on $G$.

Suppose now that $S$ is a compact semigroup such that $K(S)$ is a group. 
Then $\Pi^{*}(P(S))=\{m\}$, where $m$ is the Haar measure on $K(S)$, and Pym's result gives no information about $\Pi(P(S))$. When $S$ is a compact group (and so $K(S)=S$ ), Collins (2) gives a complete description of $\Pi(P(S))$.

Theorem 3. (Collins). Let $S$ be a compact group. Then $\Pi(P(S))$ consists of the Haar measures on maximal closed proper subgroups of $S$.

For the general case, Choy (1) has shown that the members of $\Pi(P(S))$ can be characterized in terms of the behaviour of the simple subsemigroups of $S$. In particular the measures in $\Pi(P(S))$ can be characterized in terms of their supports. The following theorem together with Theorem 3 gives a complete description of those $\mu$ in $\Pi(P(S))$ that are supported in $K(S)$.

Theorem 4. Let $S$ be a compact semigroup whose kernel $K$ is a group. Then

(i) $\Pi(P(K)) \subset \Pi(P(S))$,

(ii) if $\mu \in \Pi(P(S))$ with supp $\mu \cap K \neq \varnothing$, then supp $\mu \subset K$ and $\mu \in \Pi(P(K))$.

Proof. (i) Let $\mu \in \Pi(P(K)), v \in P(S)$. Then

$$
\text { supp } \mu v \mu=\operatorname{supp} \mu \text { supp } v \text { supp } \mu \subset K
$$

and so $\mu P(S) \mu \subset P(K)$. It follows that $\mu P(S) \mu=\mu P(K) \mu$ and therefore

by Lemma 1 .

$$
\mu \in \Pi(P(S))
$$

(ii) Let $\mu \in \Pi(P(S)), T=\operatorname{supp} \mu$, and suppose $H=T \cap K \neq \varnothing$. Then $T H \subset T \cap K=H$, and similarly $H T \subset H$, so that $H$ is an ideal of $T$. Since $T$ is simple, we have $H=T, T \subset K$. Since $\mu P(K) \mu \subset \mu P(S) \mu$, it follows from Lemma 1 that $\mu \in \Pi(P(K))$.

Let $e$ be the identity of the group $K$ and let $\delta_{e}$ be the point mass at $e$. Choy (1) shows that $\delta_{e}$ is central in $P(S)$, i.e. $\delta_{c} \mu=\mu \delta_{e}$ for each $\mu \in P(S)$, and the mapping $\Phi: P(S) \rightarrow P(K)$ defined by

$$
\Phi(\mu)=\delta_{e} \mu
$$

is a continuous homomorphism that maps $\Pi(P(S))$ onto $\Pi(P(K)) \cup\{m\}$. We have of course $\Phi(\mu)=\mu$ for each $\mu \in P(K)$. The result below shows that $\Phi(\mu)=m$ for each $\mu \in \Pi(P(S))$ that is supported outside $K$.

Proposition 5. Let $\mu \in \Pi(P(S))$ with supp $\mu \cap K=\varnothing$. Then $\delta_{e} \mu=m$ and $e$ supp $\mu=K$.

Proof. Let $T=\operatorname{supp} \mu$, so that $T$ is simple and hence is a union of subgroups of $S$. Since $e$ is central in $S$, it follows that $e T$ is a closed subgroup of $K$. Let $v$ be the Haar measure on $e T$. Since $\delta_{e} \mu$ is an idempotent measure supported on $e T$ we have $\delta_{e} \mu=v$. Hence $v \mu=\delta_{e} \mu^{2}=\delta_{e} \mu=v$ and similarly $\mu v=v$. We have $v \neq \mu$ since $T \cap K=\varnothing$, and since $\mu \in \Pi(P(S))$ it follows that $v=m$, $e T=K$.

The primitive idempotent measures on a compact group are described in terms of the maximal closed proper subgroups. Given any compact semigroup E.M.S. $\longrightarrow \mathrm{G}$ 
$S$, we recall that each subgroup of $S$ is contained in a maximal subgroup of $S$, and the maximal subgroups of $S$ are closed and pairwise disjoint (see e.g. (4), Theorem 1.1.3). Let $\mathscr{G}$ denote the family of maximal subgroups of $S$, and for each $e \in I(S)$ let $G(e)$ denote the unique maximal subgroup of $S$ that contains $e$. In the final section we shall require some results about the maximal simple subsemigroups of $S$. We recall that each simple subsemigroup of $S$ is contained in a maximal simple subsemigroup of $S$, and the maximal simple subsemigroups are closed, but need not be pairwise disjoint (see (4), p. 42). Let $\mathscr{M}$ denote the family of maximal simple subsemigroups of $S$. The next result shows that each $M \in \mathscr{M}$ is the (pairwise disjoint) union of members of $\mathscr{G}$.

Proposition 6. Let $S$ be a compact semigroup, let $M \in \mathscr{M}$ and let $e \in I(S) \cap M$. Then $G(e) \subset M$.

Proof. Write $G=G(e)$. Since $e M e$ is a group with identity $e$, we have $e M e \subset G$. Let $T$ be the subsemigroup of $S$ generated by $M$ and $G$. Since $G M G=G e M e G=G$, it follows that

$$
T=M \cup G \cup M G \cup G M \cup M G M .
$$

It is easily checked that, for each $x \in T, T x T \supset G$ and so

$$
T x T \supset T(T x T) T \supset M e M=M
$$

since $M$ is simple. Therefore $T x T=T$ for each $x \in T$ and so $T$ is simple. By maximality $T=M$, and therefore $G(e) \subset M$.

Proposition 7. Let $S$ be a compact semigroup and suppose the idempotents from distinct members of $\mathscr{M}$ commute with each other. Then the members of $\mathscr{M}$ are pairwise disjoint.

Proof. Let $M, N \in \mathscr{M}$ with $M \cap N \neq \varnothing$, and suppose that $M \neq N$. Let $e \in I(M \cap N)$ and let $f \in I(e M)$. Since $e \in N, f \in M$ we have

$$
f=e f=f e=e f e .
$$

Then $e f e$ is an idempotent in $e M e$ and so $e=e f e=f$. Thus $I(e M)=\{e\}$ and similarly $I(M e)=\{e\}$. Since $M$ is simple, it follows that $M=G(e)$, and similarly $N=G(\dot{e})$. This contradiction completes the proof.

Another sufficient condition for the members of $\mathscr{M}$ to be pairwise disjoint is that each $M \in \mathscr{M}$ should be left simple, i.e. $M e=M$ for each $e \in I(M)$ (see (4), Theorem 1.3.13); or that each $M \in \mathscr{M}$ should be right simple.

\section{The commutative case}

Throughout this section $S$ denotes a compact commutative semigroup. Thus each simple subsemigroup of $S$ is a group, and in particular the kernel $K$ of $S$ is a group. Let $e$ denote the identity of $K$ and $m$ the Haar measure on $K$. Then $I(S)$ is a compact semigroup with zero $e$, and $P(S)$ is a compact semigroup with zero $m$. The idempotent measures on $S$ are precisely the Haar measures on compact subgroups of $S$. 
Theorem 8. Let $\mu \in I(P(S))$, let $T=\operatorname{supp} \mu$ with $T \cap K=\varnothing$, and let $f$ be the identity of $T$. Then the following statements are equivalent.

(i) $\mu \in \Pi(P(S))$;

(ii) $T \in \mathscr{G}, T e=K, f \in \Pi(I(S))$.

Proof. (i) $\Rightarrow$ (ii). Let $\mu \in \Pi(P(S))$. Then $T e=K$ by Proposition 5. Let $T \subset G \in \mathscr{G}$ and let $v$ be the Haar measure on $G$. Since $v$ is the zero of $P(G)$, we have $\mu v=v, v \neq m$. Since $\mu \in \Pi(P(S))$, it follows that $\mu=v$ and $T=G$. Let $j \in I(f I(S) f)$ with $j \neq e$. Then $j f=j$ and $j G$ is a subgroup of $S$ so that $j G \subset G(j)$. Let $\rho$ be the Haar measure on $j G$. Since $\delta_{j} \mu$ is idempotent with support $j G$, we have $\delta_{j} \mu=\rho$ and so $\rho \mu=\mu$. Since $\mu \in \Pi(P(S))$, it follows that $\mu=\rho$ and so $j=f$. Therefore $f \in \Pi(I(S))$.

(ii) $\Rightarrow$ (i). Let condition (ii) hold. Let $v \in I(P(S))$ with $\mu v=v$ and let $H=$ supp $v$. Suppose first that $H \cap K \neq \varnothing$. Then $e \in H \cap K$ and $H=T H \supset T e=K$. Since $K \in \mathscr{G}$, we have $H=K, v=m$. Suppose now that $H \cap K=\varnothing$, and that $j$ is the identity of $H$. Since $f j$ is an idempotent and $T H=H$, we have $j=f j$ and so $j \in I(f I(S) f)$. Since $f \in \Pi(I(S))$, it follows that $j=f, H \subset T$. But then $\mu \nu=\mu$ and so $v=\mu$. Therefore $\mu \in \Pi(P(S))$.

Corollary 9. If $S$ has a zero, then $K=\{e\}$ and there is a one-one correspondence between $\Pi(I(S))$ and $\Pi(P(S))$ in which $j \in \Pi(I(S))$ corresponds to the Haar measure on $G(j)$.

We remark that it is easy to show by examples that the three conditions (i) $T \in \mathscr{G}$ (ii) $T e=K$ (iii) $f \in \Pi(I(S))$ are independent of each other.

Theorem 8 together with Theorems 5 and 3 gives a complete intrinsic characterization of the elements of $\Pi(P(S))$. The next result describes the structure of $\Pi(P(S))$. The simple proof below was suggested by the referee.

Theorem 10. $\Pi(P(S)) \cup\{m\}$ is a compact idempotent semigroup with $\mu \nu=m$ $(\mu \neq v)$ and with discrete topology on $\Pi(P(S))$.

Proof. $\Pi(P(S)) \cup\{m\}$ is a closed subset of $\boldsymbol{P}(S)$ by (1), Lemma 2.2. Let $\mu, v \in \Pi(P(S))$ with $\mu \neq v$. Then $\mu v$ is idempotent, and $\mu v=\mu(\mu v) \mu \in\{\mu, m\}$ as $\mu$ is primitive. Similarly, $\mu v \in\{v, m\}$. Hence $\mu v=m$. That $\Pi(P(S))$ now has the discrete topology is shown in (1), Lemma 2.3.

Given any (commutative) compact semigroup $S, I(S)$ is a compact idempotent semigroup and its kernel is a single point, i.e. $I(S)$ always has a zero element, whereas $S$ need not have a zero element.

Proposition 11. If $S$ has a zero element, then $\Pi(S)=\Pi(I(S))$.

Proof. Note that $S$ and $I(S)$ have the same zero element. Let $j \in I(S)$. It is clearly sufficient to show that $I(j S)=I(j I(S))$. Let $f \in I(j S)$. Then $f=i f \in I(j I(S))$. The other inclusion is trivial and so the proof is complete. 
Corollary 12. If $S$ has a zero element, there is a one-one correspondence between $\Pi(S)$ and $\Pi(P(S)), \Pi(S) \leftrightarrow \Pi(P(S))$. In particular,

$$
\Pi(P(S)) \leftrightarrow \Pi(P(P(S))) \text {. }
$$

Proof. Apply Corollary 9 and Proposition 11. For the final statement, recall that $P(S)$ has zero element $m$.

We can add a little to Corollary 12 by describing the maximal subgroups of $P(S)$ that support a primitive idempotent measure. Glicksberg (3) has shown that an arbitrary closed subgroup $\Gamma$ of $P(S)$ consists of the $G$-translates of Haar measure on $H$, where $G$ is a closed subgroup of $S$ and $H$ a closed subgroup of $G$. It is clear that $\Gamma$ is maximal if and only if $G$ is maximal. Given $\mu \in \Pi(P(S)$ with $\operatorname{supp} \mu \cap K=\varnothing$, it is then clear that $G(\mu)=\{\mu\}$. Given $\mu \in \Pi(P(S))$ with supp $\mu \subset K$, we see that $G(\mu)$ consists of the $K$-translates of $\mu$ and also $G(\mu)$ may be identified with $K / \operatorname{supp} \mu$.

Remarks. (1) Theorem 8 remains true (with identical proof) under the weaker hypothesis that $S$ is a compact semigroup in which each idempotent is central, and so, in particular, Theorem 8 holds for compact inverse semigroups. Theorem 10 also remains true if the idempotents of $S$ are central and $K(S)$ is commutative. Note from (1), Example 2.8 (iii) that if $S$ is the symmetric group of order 3 then $\Pi(P(S)) \cup\{m\}$ is not a subsemigroup of $P(S)$. Hence for an extension of Theorem 10 we need some restriction on $K(S)$.

(2) Suppose now that $S$ is a compact semigroup in which all the idempotents of $S$ commute with each other. Then again every simple subsemigroup of $S$ is a group and the idempotents of $P(S)$ are Haar measures on compact subgroups of $S$. In Theorem 8 we have (ii) $\Rightarrow$ (i), but (i) $\Rightarrow$ (ii) is false as the following example shows. Let $S$ be the $2 \times 2$ matrix semigroup consisting of the four matrix units, the zero matrix, the identity matrix and the matrix

$$
\left(\begin{array}{ll}
0 & 1 \\
1 & 0
\end{array}\right)
$$

It is readily verified that $S$ is a semigroup with zero in which $I(S)$ is commutative and

$$
\Gamma(I(S))=\left\{\left(\begin{array}{ll}
1 & 0 \\
0 & 0
\end{array}\right), \quad\left(\begin{array}{ll}
0 & 0 \\
0 & 1
\end{array}\right)\right\}
$$

However it is not difficult to check that the Haar measure $\mu$ on the subgroup

$$
G=\left\{\left(\begin{array}{ll}
1 & 0 \\
0 & 1
\end{array}\right), \quad\left(\begin{array}{ll}
0 & 1 \\
1 & 0
\end{array}\right)\right\}
$$

is a primitive idempotent measure. In fact $\Pi(P(S))$ consists of $\mu$ and the point masses on $\Pi(I(S))$. Note that $\Pi(P(S)) \cup\{m\}$ fails to be a subsemigroup of $P(S)$. 


\section{IDEMPOTENT MEASURES ON COMPACT SEMIGROUPS}

\section{Some non-commutative cases}

Let $S$ be an arbitrary compact semigroup and let $C \Pi(P(S))$ denote the set of central primitive idempotent measures on $S$. If the kernel $K$ of $S$ is not a group, we easily deduce from Theorem 2 that $C \Pi(P(S))=\varnothing$. For the rest of this section we suppose that $S$ is a compact semigroup whose kernel $K$ is a group. Recall that a subset $E$ of $S$ is normal if $x E=E x$ for each $x \in S$. Choy (1), Theorem 3.2 shows that the central idempotent measures on $S$ are precisely the Haar measures on compact normal subgroups of $S$. The theorem below shows that the description of $C \Pi(P(S))$ is closely related to the commutative case. We need first a simple lemma.

Lemma 13. Let $G$ be a normal subgroup of $S$ and let $j \in I(S)$. Then $j G$ is a subgroup of $S$.

Proof. We note that $j$ is central in $G$. In fact given $x \in G$, there is $y \in G$ with $j x=y j$ and then $j x=j x j$ and similarly $x j=j x j$. Let $f$ be the identity of $G$. Then $j f$ is an idempotent. Since $j G=G j$ we have $j G=j G j$ and so

$$
j G j G=j G G j=j G j=j G .
$$

Thus $j G$ is a semigroup with identity if. Given $x \in G$, we have $x^{-1} j \in j G$ and

$$
j x x^{-1} j=j f j=j f \text {. }
$$

This completes the proof.

Theorem 14. Let $\mu \in I(P(S))$, let $T=\operatorname{supp} \mu$ with $T \cap K=\varnothing$, and let $f$ be the identity of $T$. Then the following statements are equivalent.

(i) $\mu \in C \Pi(P(S))$;

(ii) $T \in \mathscr{G}, T$ is normal, $T e=K, I(f S f)=\{f, e\}$.

Proof. Argue as in Theorem 8. For the proof of (i) $\Rightarrow$ (ii) we need to use Lemma 13 above, together with the fact that if $j$ is central in $T$ then $\delta_{j} \mu=\mu \delta_{j}$ and so $\delta_{j} \mu$ is idempotent.

Theorem 14 describes the elements of $C \Pi(P(S))$ that are supported outside the kernel $K$. The elements of $C \Pi(P(S))$ that are supported in the kernel are precisely the Haar measures on the maximal closed subgroups of $K$ that are normal in $S$.

Theorem 15. $C \Pi(P(S)) \cup\{m\}$ is a compact idempotent semigroup with $\mu v=m(\mu \neq v)$ and with discrete topology on $\Pi(P(S))$.

Proof. Argue as in Theorem 10.

Now let $S$ be a compact semigroup (whose kernel $K$ is a group) such that

$$
M, N \in \mathscr{M}, M \neq N, j \in I(M) \Rightarrow j N=N j \text {. }
$$


Note that $j$ is then central in $N$, and, by Proposition 7, the members of $\mathscr{M}$ are pairwise disjoint. Thus each simple subsemigroup of $S$ is contained in a unique maximal simple subsemigroup. In particular, the support of any idempotent measure on $S$ is contained in a unique maximal simple subsemigroup of $S$. When $M \in \mathscr{M}$ is a group with $M \cap K=\varnothing$ we make a convenient abuse of notation by writing $\Pi(P(M))$ for the set consisting of the Haar measure on $M$.

Theorem 16. Let $S$ satisfy $\left(^{*}\right)$, let $\mu \in I(P(S))$ and let $T=\operatorname{supp} \mu$ with $T \subset M \in \mathscr{M}, T \cap K=\varnothing$. Then the following statements are equivalent.

(i) $\mu \in \Pi(P(S))$;

(ii) $\mu \in \Pi(P(M)), T e=K, I(j S j)=\{j, e\}$ for each $j \in I(T)$.

Proof. (i) $\Rightarrow($ ii). Let $\mu \in \Pi(P(S))$. Then $\mu \in \Pi(P(M))$, and, by Proposition 5, $T e=K$. Let $j \in I(T), f \in I(j S j)$ and suppose $f \neq e, f \notin M$. Then $f$ is central in $T$ and so $\mu \delta_{f}=\delta_{f} \mu$. Let $\rho=\mu \delta_{f}$ and then $\rho^{2}=\rho, \mu \rho=\rho \mu=\rho$. Therefore $f=j f \in \operatorname{supp} \rho$. But $\mu \in \Pi(P(S))$ gives $\rho=m$ or $\rho=\mu$. This contradiction shows that $I(j S j) \subset M \cup\{e\}$. If $f \in M$, then $f=j f j \in j M j=G(j)$ and so $f=j$.

(ii) $\Rightarrow$ (i). Let condition (ii) hold, let $v \in I(P(S))$ with $\mu v=v \mu=v$ and let $R=\operatorname{supp} v$. Then $R$ is simple and there is $N \in \mathscr{M}$ with $R \subset N$. If $N=K$, then

$$
R=T R \supset T e=K
$$

and so $v=m$. If $N=M$, then $v=\mu$ since $\mu \in \Pi(P(M))$. Suppose finally that $N \cap M \cap K=\varnothing$. Let $j \in I(T)$. Then $j$ is central in $R$, and since $R T=T R=R$, it follows that there is an idempotent in $R \cap j S j$. This contradiction completes the proof.

As an illustration of Theorem 16, let $R$ be the disjoint union of a family $\left\{S_{\lambda}: \lambda \in \Lambda\right\}$ of compact simple semigroups and let the union of the topologies on the $S_{\lambda}$ be a base for a topology on $R$. Then $R$ is locally compact. Now let $S=R \cup\{\theta\}$ with the topology of the one point compactification of $R$. Extend the multiplications on the $S_{\lambda}$ to a multiplication on $S$ by defining all new products to be $\theta$. It is now easily checked that $S$ is a compact semigroup. A simple application of Theorem 16 gives

$$
\Pi(P(S))=\cup\left\{\Pi\left(P\left(S_{\lambda}\right)\right): \lambda \in \Lambda\right\}=\cup\left\{K\left(P\left(S_{\lambda}\right)\right): \lambda \in \Lambda\right\} .
$$

The results of this paper indicate that the size of $\Pi(P(S))$ reflects the degree of non-commutativity of $S$. As the extreme example, let $S$ be a compact space with multiplication

$$
x y=x \quad(x, y \in S) .
$$

Then $S$ is a compact simple semigroup and $P(S)$ has multiplication

$$
\mu v=\mu \quad(\mu, v \in P(S)) .
$$

Therefore $\Pi(P(S))=P(S)$. 
IDEMPOTENT MEASURES ON COMPACT SEMIGROUPS 103

\section{REFERENCES}

(1) S. T. L. ChoY, Idempotent measures on compact semigroups, Proc. London Math. Soc., to appear.

(2) H. S. Collins, Primitive idempotents in the semigroup of measures, Duke Math. J. 27 (1960), 397-400.

(3) I. GLicksBerg, Convolution semigroups of measures, Pacific J. Math. 9 (1959), 51-67.

(4) A. B. Paalman de Miranda, Topological semigroups, Math. Centrum, Amsterdam (1964).

(5) J. S. Pym, Idempotent measures on semigroups, Pacific J. Math. 12 (1962), 685-698.

KING'S COLlege

UNIVERSITY OF ABERDEEN 